\title{
Blühdorn, Ingolfur, Felix Butzlaff, Michael Deflorian, Daniel Hausknost, und Mirijam Mock (2019): Nachhaltige Nicht-Nachhaltigkeit. Warum die ökologische Transformation der Gesellschaft nicht stattfindet
}

\author{
Bielefeld: transcript Verlag. 330 Seiten. 19,90€
}

\section{Hubertus Buchstein}

Online publiziert: 20. Juli 2020

(C) Der/die Autor(en) 2020

Ingolfur Blühdorn hat sich in der Vergangenheit einen Namen als ebenso notorischer wie scharfsinniger Kritiker von Demokratisierungsideen und ökologischen Modernisierungsprogrammen gemacht. Sein neues Buch, zu dem auch vier seiner MitarbeiterInnen aus Wien mit eigenen Kapiteln beigetragen haben, bietet eine in Teilen wiederholende Zusammenfassung vorheriger Arbeiten und versucht eine ,,gesellschaftsdiagnostische und -theoretische“ (S. 314) Ausweitung seiner bisherigen kritischen Einwürfe. Laut Blühdorn dokumentiert das Buch die Entwicklung seiner Überlegungen zu einem eigenständigen ,sozialwissenschaftlichen Forschungsansatz“ (S. 11), zu einem „Konzept der Gesellschaft der Nicht-Nachhaltigkeit“ (S. 67).

Das Buch besteht aus neun Kapiteln. Vier stammen aus der Tastatur von Blühdorn. Er legt darin eingangs die für das Buch titelgebende Grundthese ausführlich dar und schließt den Band mit scharfer Kritik an gegenwärtigen demokratietheoretischen Diskursen ab. Den Co-AutorInnen bleibt es vorbehalten, innerhalb des dadurch gesetzten Rahmens diese Generalthese für die Bereiche staatliche Handlungskompetenz, Zivilgesellschaft, politische Parteien, gesellschaftlicher Wertewandel sowie individuelles Konsumverhalten zu illustrieren und zu plausibilisieren.

Die Generalthese lässt sich in wenigen Worten zusammenfassen: Die gegenwärtige moderne Gesellschaft ist eine Gesellschaft der nachhaltigen Nicht-Nachhaltigkeit. Das emanzipatorische Potenzial der bürgerlichen Aufklärung sowie ihre Aktualisierung in der linken Protestbewegung der 1960er-Jahre habe sich zu einer „Emanzipation zweiter Ordnung“ (S. 103) fortentwickelt. Damit wurde der ursprünglich einmal avisierte Auszug aus der selbstverschuldeten Unmündigkeit ersetzt durch die „Befreiung aus der Mündigkeit“ (S. 103) und die Anpassung an die Gegeben-

\footnotetext{
H. Buchstein $(\bowtie)$

Institut für Politik- und Kommunikationswissenschaft, Universität Greifswald, Greifswald, Deutschland

E-Mail: buchstei@uni-greifswald.de
} 
heiten einer ressourcenverbrauchenden Konsumgesellschaft. Stellenweise lesen sich diese Ausführungen von Blühdorn in ihrem suggestiven Duktus wie ein Herbert Marcuse 2.0.

Die von Blühdorn an dieser Stelle intendierte argumentative Pointe hängt an der von ihm vorgenommenen kategorialen Verklammerung des Emanzipationsbegriffs an solche Werte wie größtmögliche individuelle Freiheit, vielseitige Selbstverwirklichung sowie einem konsum- und erlebnisorientierten Lebensstil. Würde man hingegen den Emanzipationsbegriff inhaltlich anders füllen - sei es mit kollektiver Rationalität oder individueller Autarkie - ließen sich die in dem Buch geschilderten Berichte aus dem konsumorientierten Alltag mit grünen Zertifikaten nicht primär als die destruktive Fortsetzung von Emanzipationsimpulsen einordnen, sondern sie würden unmissverständlich als regressive Elemente bewertet werden.

Doch auf eine solche begriffliche Umstellung wollen (S. 119-120) und können sich die AutorInnen des Buches nicht einlassen. Denn sie sehen in der Kultur des Emanzipationsstrebens eine zentrale Ursache für das Misslingen aller bisherigen Versuche, moderne Gesellschaften in Richtung ökologischer Transformation umzusteuern. Dies gilt ihnen zufolge auch und gerade für solche Gesellschaften wie die der Bundesrepublik, in der große Teile der politischen Öffentlichkeit den Wert der ökologischen Nachhaltigkeit stolz vor sich hertragen. Nun ist es eine längst bekannte Tatsache, dass der ökologische Fußabdruck von vielen, die sich für ökologische Politik einsetzen, dessen ungeachtet vergleichsweise groß ist. In dem Buch wird für diese Menschen der ansonsten dem Strafrecht vorbehaltene Ausdruck „Täter“ (S. 22) verwendet.

Für die AutorInnen liegt in einem solchen Verhalten weniger ein Widerspruch zwischen hohem moralischen Anspruch und den Niederungen der alltäglichen Praxis, sondern steckt dahinter System. Denn eine zweite Generalthese des Buches lautet, dass die ökologische Nicht-Nachhaltigkeit moderner Gesellschaften nicht das Resultat von zu geringen Bemühungen oder zu starken Widerständen in der Gesellschaft sei, sondern selbst zu einem inhärenten Bestandteil des gegenwärtigen Nachhaltigkeits- und Transformationsdiskurses geworden ist. Sowohl die Umweltsoziologie wie auch die Transformationsforschung baue eifrig mit an der Hegemonie eines grün-modernen Wohlfühlnarrativs, welches das tatsächliche ökologische Versagen in unserer Gegenwart kaschiere. Zur Charakterisierung dieses Zusammenhanges verwendet Blühdorn den bei Jean Baudrillard entliehenen Ausdruck der tief greifenden „Komplizenschaft“ (S. 16, 316) und geht sogar noch einen Schritt weiter, indem er den Verdacht äußert, dass der Rechtspopulismus ,eine dreckige Arbeit übernimmt, die das gepflegte Bürgertum zwar weit von sich weist, die ihm aber doch unverzichtbar ist" (S. 18).

An einer Stelle im Buch heißt es lakonisch: „Eine Begrenzung und Regulierung der Freiheit wäre [...] das Gebot der Stunde“ (S. 114). Darüber hätte man nun gern mehr und Konkreteres erfahren. Stattdessen sehen sich Blühdorn et al. gleichsam präventiv dazu veranlasst, sich gegen den Vorwurf zu erwehren, ihre Thesen seien zu pessimistisch und politisch unproduktiv. Zu Recht kontern sie solche Vorwürfe mit dem Hinweis, dass die Aufgabe von SozialwissenschaftlerInnen die gründliche und nüchterne Analyse sei und nicht die des „Hoffnungsmachers“(S. 317). Aber wie gründlich und überzeugend sind die vorgelegten Analysen? Diesbezüglich möchte 
ich Zweifel anmelden. Und zwar nicht, weil ich optimistischer als die AutorInnen bin, sondern weil die Argumentationen der einzelnen Kapitel in sozialtheoretischer Hinsicht kein kohärentes gesellschaftsdiagnostisches Gesamtbild ergeben.

Oberflächlich gesehen fügen sich die neun Kapitel mit ihren diversen internen Querverweisen zu einer bestätigenden Illustration der Grundthesen. Denn auch in methodischer Hinsicht folgen sie alle einem Muster. Sie referieren, interpretieren, analysieren, diskutieren oder kritisieren als einschlägig erachtete Literatur zu den thematischen Schwerpunkten der Buchkapitel und changieren dabei zwischen Sekundär- und Tertiäranalysen und -interpretationen. Das gelingt nicht immer gleich gut. So führt Blühdorn beispielsweise als Beleg für die Gesinnung einer Emanzipation zweiter Ordnung an, dass Verbote und eine $\mathrm{CO}_{2}$-Steuer auf breite Ablehnung in der Bevölkerung stoßen (S. 108). Er belässt es bei diesem pauschalen Hinweis, ohne näher darauf einzugehen, ob und wie sich die Zustimmung zu einer solchen Steuer beispielsweise verändert hat, wie dies von ihm im besonders kritisch beäugten grünen Milieu gesehen wird oder wie die diesbezüglich mittlerweile auf den Weg gebrachte Gesetzgebung in der Bundesrepublik zu bewerten ist. Ein anderes Beispiel ist die mehrfach im Buch zu lesende Rede von einem „hegemonialen Neoliberalismus" (S. 228), ohne dabei auf die in der neueren Kapitalismusforschung herausgestellten Differenzen zwischen verschiedenen Ländern einzugehen. Dies sind nur zwei Beispiele von vielen für die empirische Grobkörnigkeit des Buches. Darin kommen auch keine eigenen empirischen Analysen zu Wort, sondern es wird aus der Literatur oder medialen Berichten das selektiv herausgegriffen, was als Bestätigung der eigenen Thesen angesehen wird.

Diese Methode der selektiven Montage weist auf ein tiefer liegendes methodologisches Problem des „Forschungsprogramm(s)“ (S. 11) hin. Über den ganzen Band verteilt machen die AutorInnen Anleihen bei Phänomenbeschreibungen und Argumentationen, die aus disparaten und nicht leicht miteinander zu vereinbarenden soziologischen Theorieansätzen stammen. So finden sich im Kapitel über das individuelle Konsumverhalten gut begründete Anleihen aus der Praxistheorie (S. 235-241), denen ebenso einleuchtende Rational-Choice-Theoreme im Kapitel über politische Parteien (S. 183, 195) gegenüberstehen. Verstreut finden sich in dem Buch dezidiert kapitalismustheoretische Aussagen (S. 293) neben diversen Reminiszenzen an die funktionalistische Sprache der Systemtheorie (S. 88, 93-94, 307). Dann aber wieder verfällt das Buch höchst un-luhmannesk in die Tonlage drängender Moralisierungen (S. 18, 23). Des Weiteren finden sich ins Leere laufende Anschlüsse an Reckwitz' Singularitätentheorie (S. 99-100), sozial-konstruktivistische Bekenntnisse (S. 305-306), um kurz darauf mit einer im Kontext des zuvor aufgebauten Theoriedesigns naiv anmutenden Rede von den bedrohlichen ,faktischen Entwicklungen“" (S. 314) konfrontiert zu werden.

Mehrfach wird in dem Buch am Rande auf Luhmanns für die Thematik absolut einschlägige Buch „Ökologische Kommunikation“ aus dem Jahre 1986 verwiesen. Ein so sehr in Paradoxien verliebter Autor wie Luhmann hätte aus seiner Perspektive vermutlich ebenfalls gern eine Gesellschaftstheorie der nachhaltigen NichtNachhaltigkeit formuliert. Warum hätte es nicht genügt, die Grundthesen des Buches mit Rekurs allein auf Luhmann durchzubuchstabieren? Eine solche Frage lieBe sich auch aus Sicht von marxistischen Kapitalismustheorien oder von Theorien 
kollektiven Handelns des Rational-Choice-Ansatzes stellen. Die in dem Buch zum Ausdruck gelangende Theorievielfalt mündet in keinem produktiven Eklektizismus und auch in keiner hohen sozialtheoretischen Anschlussfähigkeit, sondern erweckt eher den Eindruck der Beliebigkeit. Dazu passt, dass der Hauptautor an einigen Stellen von sich aus Rückzugsmanöver vornimmt, indem er den Status der vorgestellten Überlegungen bescheidener als „Interpretationsvorschlag“ (S. 120) oder als „Plausibilitätsangebot“" (S. 120) bezeichnet.

Die Grundthesen des Buches sind so umfassend wie zugleich vage, steil und provozierend. Deshalb lohnt die Lektüre unbedingt. Aber möglicherweise lassen sich die Thesen des Buches besser in einem anderen literarischen Genre bearbeiten als dem mit dezidiert sozialwissenschaftlichen Anspruch auftretenden Sammelband. Um die beabsichtigten ,,irritierenden“ (S. 21) Effekte bei LeserInnen zu erzielen und um ,Dimensionen sichtbar zu machen, die bei vielen anderen Ansätzen im Dunkeln bleiben“ (S. 120), hätte vielleicht der sozialphilosophische Essay à la Horkheimer/ Adorno oder der flotte Essay à la Enzensberger eine angemessenere Form geboten.

Funding Open Access funding provided by Projekt DEAL.

Open Access Dieser Artikel wird unter der Creative Commons Namensnennung 4.0 International Lizenz veröffentlicht, welche die Nutzung, Vervielfältigung, Bearbeitung, Verbreitung und Wiedergabe in jeglichem Medium und Format erlaubt, sofern Sie den/die ursprünglichen Autor(en) und die Quelle ordnungsgemäß nennen, einen Link zur Creative Commons Lizenz beifügen und angeben, ob Änderungen vorgenommen wurden.

Die in diesem Artikel enthaltenen Bilder und sonstiges Drittmaterial unterliegen ebenfalls der genannten Creative Commons Lizenz, sofern sich aus der Abbildungslegende nichts anderes ergibt. Sofern das betreffende Material nicht unter der genannten Creative Commons Lizenz steht und die betreffende Handlung nicht nach gesetzlichen Vorschriften erlaubt ist, ist für die oben aufgeführten Weiterverwendungen des Materials die Einwilligung des jeweiligen Rechteinhabers einzuholen.

Weitere Details zur Lizenz entnehmen Sie bitte der Lizenzinformation auf http://creativecommons.org/ licenses/by/4.0/deed.de. 